\title{
Critical behavior of the spontaneous polarization and the dielectric susceptibility close to the cubic-tetragonal transition in $\mathrm{BaTiO}_{3}$
}

\author{
H. Yurtseven*,* and F. Oğuz ${ }^{\dagger}$ \\ *Department of Physics, Faculty of Arts and Sciences \\ Middle East Technical University, 06531 Ankara, Turkey \\ $\dagger^{\dagger}$ Micro and Nanotechnology Program \\ Graduate School of Natural Applied Sciences \\ Middle East Technical University, 06800 Ankara, Turkey \\ thamit@metu.edu.tr
}

Received 26 June 2015; Revised 27 July 2015; Accepted 4 August 2015; Published 23 September 2015

\begin{abstract}
Using Landau mean field model, the spontaneous polarization and the dielectric susceptibility are analyzed as functions of temperature and pressure close to the cubic-tetragonal (ferroelectric-paraelectric) transition in $\mathrm{BaTiO}_{3}$. From the analysis of the dielectric susceptibility and the spontaneous polarization, the critical exponents are deduced in the classical and quantum limits for $\mathrm{BaTiO}_{3}$. From the critical behavior of the dielectric susceptibility, the spontaneous polarization can be described for the ferroelectric-paraelectric (cubic to tetragonal) transition between 4 and $8 \mathrm{GPa}$ at constant temperatures of 0 to $200 \mathrm{~K}$ in $\mathrm{BaTiO}_{3}$ within the Landau mean field model given here.
\end{abstract}

Keywords: Dielectric susceptibility; spontaneous polarization; ferroelectric-paraelectric transition; Landau mean field model; $\mathrm{BaTiO}_{3}$.

\section{Introduction}

The dielectric properties of $\mathrm{BaTiO}_{3}$ as a well-known ferroelectric material ${ }^{1}$ have been studied at various hydrostatic pressures and temperatures. ${ }^{2}$ It exhibits successive transitions between cubic and tetragonal $(\mathrm{C}-\mathrm{T})$ phases (paraelectricferroelectric), between tetragonal and orthorhombic (T-O) phases (ferroelectric-ferroelectric) and between orthorhombic-rhombohedral $(\mathrm{O}-\mathrm{R})$ phases (ferroelectric-ferroelectric) which have been studied experimentally, as shown in the T-P phase diagrams. ${ }^{2,3}$ From the dielectric study of $\mathrm{BaTiO}_{3}$, it was found that the cubic-tetragonal $(\mathrm{C}-\mathrm{T})$ transition temperature in particular decreased linearly with pressure in the range of 1 bar to $3 \mathrm{GPa}$, as also pointed out previously. ${ }^{3}$ Above $3 \mathrm{GPa}$, there occurs the critical point at which the firstand second-order transitions cross over as suggested in an earlier study. ${ }^{2}$ For the hexagonal-orthorhombic $(\mathrm{H}-\mathrm{O})$ phase transition in hexagonal $\mathrm{BaTiO}_{3}\left(h-\mathrm{BaTiO}_{3}\right)$, the critical pressure was obtained as $3.4 \mathrm{GPa} .{ }^{4}$ Its specific heat can be compared with that of perovskite $\mathrm{BaTiO}_{3}\left(p-\mathrm{BaTiO}_{3}\right)$ with a similar Debye temperature, ${ }^{5}$ as also pointed out previously. ${ }^{3}$ At room temperature, $\mathrm{BaTiO}_{3}$ has a tetragonal perovskite structure with the point group $\mathrm{C}_{4 \mathrm{~V}}$. As the temperature increases up to the Curie temperature $(\sim 400 \mathrm{~K})$, it undergoes the cubic phase with the point group $\mathrm{O}_{\mathrm{h}} .{ }^{1}$ The Raman spectroscopy has been used to investigate the tetragonal phase in $\mathrm{BaTiO}_{3}$ in some earlier studies. ${ }^{6-9}$ Studies on infrared reflectivity, ${ }^{10}$ Raman $^{10,11}$ and light scattering ${ }^{12}$ have focused on the soft modes in $\mathrm{BaTiO}_{3}$.

Thin films of barium titanate using X-ray diffraction ${ }^{13}$ and Raman spectroscopy ${ }^{13,14}$ have been studied to investigate the ferroelectric-paraelectric transition. Recently, ferroelectric transition in barium titanate nanoparticles has been studied using NMR spectra. ${ }^{15}$ We have also studied soft modes in ferroelectric ${ }^{16}$ and hexagonal $\mathrm{BaTiO}_{3} \cdot{ }^{17}$ We have calculated the damping constant for the tetragonal Raman mode of $\mathrm{BaTiO}_{3} .{ }^{18}$

In regard to the dielectric properties of barium titanate, continued existence of a permanent polarization above the Curie temperature was reported many years ago and above $120^{\circ}$, the dielectric susceptibility obeys the Curie-Weiss law, ${ }^{19}$ as also studied in the cubic paraelectric phase of this material. ${ }^{2}$ It has been reported that porosity and pore size effect on the dielectric properties of $\mathrm{BaTiO}_{3}$ ceramic and that the dielectric susceptibility decreases abruptly as the porosity increases. ${ }^{20}$ It has also been observed experimentally that the dielectric susceptibility increased with increasing the amount of dopant for $\mathrm{Ba}_{1-x} \mathrm{Ce}_{x} \mathrm{TiO}_{3}$ compounds. ${ }^{21}$

In order to investigate the temperature and pressure dependence of the dielectric constant (dielectric susceptibility) and the spontaneous polarization close to the phase transitions in $\mathrm{BaTiO}_{3}$, the Landau mean field models have been employed. ${ }^{12,13,15}$ By expanding the free energy in terms of

This is an Open Access article published by World Scientific Publishing Company. It is distributed under the terms of the Creative Commons Attribution 3.0 (CC-BY) License. Further distribution of this work is permitted, provided the original work is properly cited. 
the order parameter (spontaneous polarization $P$ ), the temperature dependence of the $P$ and the dielectric susceptibility $\chi$ (dielectric constant $\epsilon$ ) can be obtained by determining the coefficients in the free energy expansion. The temperature dependence of the bilinear coupling constant (order parameter) of the soft-optic and acoustic mode in hexagonal barium titanate has been analyzed using the experimental data ${ }^{12}$ according to the prediction of the mean field theory. Experimental measurements for variation of the order parameters (wavenumber position and width) with temperature, have been interpreted using Landau-Devonshire thermodynamic formalism for bulk perovskites in the case of thin films of $\mathrm{BaTiO}_{3} .{ }^{13}$ For the $\mathrm{C}-\mathrm{T}$ phase transition of $\mathrm{BaTiO}_{3}$, the quadrupole coupling constant (order parameter) has been analyzed as a function of temperature using the experimental measurements for barium titanate nanoparticles on the basis of the Landau expansion of the free-energy density. ${ }^{15}$ Very recently, we have analyzed ${ }^{22}$ the temperature dependence of the polarization and the dielectric susceptibility near the $\mathrm{C}-\mathrm{T}$ transition by using the experimental data for the lattice mode (order parameter) of thin films in $\mathrm{BaTiO}_{3}$ according to the Landau phenomenological theory.

In this study, by presenting the Landau mean field model we predict the temperature and pressure dependence of the spontaneous polarization from that dependence of the dielectric susceptibility for the ferroelectric-paraelectric $(\mathrm{C}-\mathrm{T})$ transition in $\mathrm{BaTiO}_{3}$. Experimental data are used to analyze the critical behavior of the dielectric susceptibility, as also analyzed previously ${ }^{3}$ and the critical exponents are deduced for the spontaneous polarization in the classical and quantum limits for the ferroelectric-paraelectric transition in $\mathrm{BaTiO}_{3}$.

Below, in Sec. 2, we introduce the Landau mean field model and give our calculations and results. We discuss our results in Sec. 3. Conclusions are given in Sec. 4.

\section{Calculations and Results}

We calculate here the spontaneous polarization as functions of temperature and pressure from the dielectric susceptibility close to the cubic tetragonal transitions in $\mathrm{BaTiO}_{3}$ which exhibits first-order transition. However, above $3 \mathrm{GPa}$, the first-order and second-order transitions crossover at the critical point in $\mathrm{BaTiO}_{3}{ }^{2}$ Our analysis of the experimental data ${ }^{3}$ using Landau phenomenological theory is based on both firstorder and second-order transitions which occur in $\mathrm{BaTiO}_{3}$.

In the Landau phenomenological theory, the free energy of a ferroelectric material can be expressed in terms of the spontaneous polarization $P$ as

$$
F=a_{0}+a_{2} P^{2}+a_{4} P^{4}
$$

where

$$
a_{2}=\alpha\left(T-T_{\mathrm{C}}\right)
$$

with the $\alpha, a_{0}$ and $a_{4}$ as constants. $T_{\mathrm{C}}$ is the Curie temperature.

In the paraelectric phase $\left(T>T_{\mathrm{C}}\right)$, we have $P=0$ whereas in the ferroelectric phase $\left(T<T_{\mathrm{C}}\right), P \neq 0$.

From the minimization of ferroelectric free energy, $\frac{\partial F}{\partial P}=0$, we have

$$
2 P\left(a_{2}+2 a_{4} P^{2}\right)=0 .
$$

This gives the solutions,

$$
P=0 \quad \text { (paraelectric phase) }
$$

and

$$
P^{2}=-a_{2} / 2 a_{4} \quad(\text { ferroelectric phase }) .
$$

Also, the temperature dependence of the inverse susceptibility $\left(\chi^{-1}\right)$ can be derived from Eq. (1) by defining

$$
\chi^{-1}=\frac{\partial^{2} F}{\partial P^{2}}
$$

which gives

$$
\chi^{-1}=2 a_{2}+12 a_{4} P^{2} .
$$

When $P=0$ in the paraelectric phase $\left(T>T_{\mathrm{C}}\right)$, we obtain

$$
\chi^{-1}=2 a_{2}
$$

so, the temperature dependence of the inverse susceptibility is given by

$$
\chi^{-1}=2 \alpha\left(T-T_{\mathrm{C}}\right) .
$$

In the ferroelectric phase $\left(T<T_{\mathrm{C}}\right)$ from Eq. (5) by using Eq. (2), we obtain

$$
\chi^{-1}=2 \alpha\left(T-T_{c}\right)+12 a_{4} P^{2} .
$$

Thus, from the mean-field theory, the value of the critical exponent is deduced as $\gamma_{T}=1$ for the inverse susceptibility according to the power law formula in the paraelectric phase,

$$
\chi^{-1} \propto\left(T-T_{\mathrm{C}}\right)^{\gamma_{T}} .
$$

Also, in the ferroelectric phase, this theory predicts that the critical exponent $\beta$ for the polarization is $\beta=1 / 2$ according to

$$
P=c\left(T-T_{\mathrm{C}}\right)^{\beta},
$$

where $c$ is the amplitude.

By analyzing the experimental data for the inverse dielectric susceptibility, Ishidate et al. ${ }^{3}$ obtained the values of $\gamma_{T}=1.2$ at $4 \mathrm{GPa}$ for the paraelectric phase $\left(T>T_{\mathrm{C}}\right)$ in the classical limit $\left(T_{\mathrm{C}}=212 \mathrm{~K}\right)$ for the $\mathrm{C}-\mathrm{T}$ transition in $\mathrm{BaTiO}_{3}$. Their value was $\gamma_{T}=1.7$ at $7 \mathrm{GPa}$ for the paraelectric phase $\left(T>T_{\mathrm{C}}\right)$ in the quantum limit $\left(T_{\mathrm{C}}=0 \mathrm{~K}\right)$. For both analyses, they used the power law formula.

$$
\chi^{-1}=b\left(T-T_{\mathrm{C}}\right)^{\gamma_{T}}
$$

with the critical exponent $\gamma_{T}$ for the dielectric susceptibility $\chi$ and the amplitude $b$. 
Using the temperature dependence of the inverse susceptibility $\chi^{-1}$ (Eq. (8)) and the spontaneous polarization $P$ (Eq. (10)) for the ferroelectric phase, we find through Eq. (11),

$$
b\left(T-T_{\mathrm{C}}\right)^{\gamma_{T}-1}=2 \alpha+12 a_{4} c^{2}\left(T-T_{\mathrm{C}}\right)^{2 \beta-1} .
$$

This then gives

$$
\left(T-T_{\mathrm{C}}\right)^{\gamma_{T}-1} \sim\left(T-T_{\mathrm{C}}\right)^{2 \beta-1}
$$

with

$$
\beta_{T}=\gamma_{T} / 2 \text {. }
$$

In the classical limit $\gamma_{T}=1$ and $\beta=1 / 2$, whereas in the quantum limit $\gamma_{T}=2$ and $\beta=1$ (Eq. (14)). For the paraelectric phase at $4 \mathrm{GPa}\left(T_{\mathrm{C}}=212 \mathrm{~K}\right)$, we then find $\beta=$ $1.2 / 2=0.6$ since $\gamma_{T}=1.2$ and at $7 \mathrm{GPa}\left(T_{\mathrm{C}}=212 \mathrm{~K}\right) \beta=$ $1.7 / 2=0.85$ with $\gamma_{T}=1.7 .^{3}$

From the analysis of their experimental results for the inverse dielectric susceptibility, Ishidate et al. ${ }^{3}$ obtained $\gamma_{T}$ values at various pressures for $\mathrm{BaTiO}_{3}$, as given in Table 1. In this table, we also give our $\beta$ values according to Eq. (14).

The pressure dependence of the inverse dielectric susceptibility $\chi$ and also the spontaneous polarization $P$ at constant temperature can be obtained close to the transition point in $\mathrm{BaTiO}_{3}$. By considering in the free energy expansion (Eq. (1))

$$
a_{2}=a_{20}\left(p-p_{c}\right),
$$

where $a_{20}$ is constant and $p_{c}$ is the critical pressure from Eq. (6) through Eq. (15) in the paraelectric phase $(P=0)$, the pressure dependence of the inverse susceptibility can be written as

$$
\chi^{-1}=2 a_{20}\left(p-p_{c}\right) .
$$

In the ferroelectric phase, using Eq. (5) the inverse susceptibility can be obtained at various pressures as follows:

$$
\chi^{-1}=2 a_{20}\left(p-p_{c}\right)+12 a_{4} P^{2} .
$$

Thus, from the mean-field theory, the critical exponent is $\gamma_{p}=$ $\gamma_{T}=1$ for the inverse susceptibility in the classical limit according to the power-law formula

$$
\chi^{-1} \propto\left(p-p_{c}\right)^{\gamma_{p}}
$$

in the paraelectric phase.

Table 1. Values of the critical exponents $\gamma_{T}$ and $\beta_{T}$ for the dielectric susceptibility $\chi$ and the spontaneous polarization $P$, respectively, at various pressures close to the $\mathrm{C}-\mathrm{T}$ transition in $\mathrm{BaTiO}_{3}$.

\begin{tabular}{lcc}
\hline$P(\mathrm{GPa})$ & $\gamma_{T}$ & $\beta_{T}$ \\
\hline 2.99 & 0.99 & 0.50 \\
3.99 & 1.20 & 0.60 \\
4.98 & 1.70 & 0.85 \\
5.97 & 1.49 & 0.75 \\
6.98 & 1.70 & 0.85 \\
7.97 & 1.20 & 0.60 \\
\hline
\end{tabular}

In the ferroelectric phase, the mean-field theory predicts that the critical exponent for the polarization is $\beta_{p}=1 / 2$ also in the classical limit using the power-law formula

$$
P=d\left(p-p_{c}\right)^{\beta_{p}},
$$

where $d$ is constant on the basis of

$$
\chi^{-1}=e\left(p-p_{c}\right)^{\gamma_{p}},
$$

where $e$ is constant. This can be obtained easily from the power-law formula through Eq. (17) and using Eq. (19),

$$
e\left(p-p_{c}\right)^{\gamma_{p}-1}=2 a_{20}+12 a_{4} d^{2}\left(p-p_{c}\right)^{2 \beta_{p}-1}
$$

which then gives

$$
\left(p-p_{c}\right)^{\gamma_{p}-1} \sim\left(p-p_{c}\right)^{2 \beta_{p}-1}
$$

with

$$
\beta_{p}=\gamma_{p} / 2
$$

the same result as Eq. (14).

\section{Discussion}

We studied the temperature dependence of the spontaneous polarization and dielectric susceptibility (dielectric constant) close to the ferroelectric-paraelectric transition in $\mathrm{BaTiO}_{3}$ using the mean field theory. Expressions for the spontaneous polarization (ferroelectric phase) and the dielectric susceptibility (ferroelectric and paraelectric phases) were derived. The critical behavior of the dielectric susceptibility and the spontaneous polarization was described by a power-law formula with the critical exponents $\gamma_{T}$ and $\beta_{T}$, respectively, close to the ferroelectric-paraelectric transition in $\mathrm{BaTiO}_{3}$. It was found that $\beta_{T}=\gamma_{T / 2}$, and using the $\gamma_{T}$ values as obtained from the analysis of Ishidate et al., ${ }^{3}$ we extracted the $\beta_{T}$ values for the spontaneous polarization in the ferroelectric phase of $\mathrm{BaTiO}_{3}$. Those values of the critical exponents were obtained between $\gamma_{T}=1, \beta_{T}=1 / 2$ (classical limit) and $\gamma_{T}=2, \beta_{T}=$ 1 (quantum limit) for the ferroelectric-paraelectric transition in $\mathrm{BaTiO}_{3}$. From the analysis of the experimental measurements of the dielectric susceptibility, values of the critical exponent were obtained as $\gamma_{T}=1.2$ (at $4 \mathrm{GPa}$ ) and $\gamma_{T}=1.7$ (at $7 \mathrm{GPa}$ ) by Ishidate et $a .^{3}$ in the classical and quantum limits, respectively, for $\mathrm{BaTiO}_{3}$. This led us to extract the values of $\beta_{T}=0.60$ (classical limit) and $\beta_{T}=0.85$ (quantum limit) according to Eq. (14) as given in Table 1 .

We extended this study to the pressure dependence of the dielectric susceptibility $\chi$ and the spontaneous polarization $P$ at constant temperatures of 100 and $200 \mathrm{~K}$ using the experimental data $^{3}$ for the paraelectric-ferroelectric transition in $\mathrm{BaTiO}_{3}$. Using the mean field theory by expanding the free energy in terms of the order parameter (spontaneous polarization), we obtained the pressure dependencies of both spontaneous polarization $P$ and the dielectric susceptibility $\chi$, as we obtained for the temperature dependence of $P$ and $\chi$. 
From the pressure dependence of the dielectric susceptibility $\chi$ (Eq. (20)), Ishidate $e t a l .^{3}$ concluded that the critical exponent value of $\gamma_{p}=1$ as predicted from the quantum theory, is valid at constant temperatures of 100 and $200 \mathrm{~K}$ in the cubic (paraelectric) phase of $\mathrm{BaTiO}_{3}$. This implies from the pressure dependence of the spontaneous polarization $P$ (Eq. (19)), the critical index $\beta_{p}=1 / 2$ (Eq. (23)) is also valid in the quantum limit. This is due to the fact that in the quantum limit, the critical temperature $T_{c}$ depends on the interaction parameter $S$ (an external parameter such as pressure or chemical composition) ${ }^{23,24}$ according to

$$
T_{c}(S) \propto\left(S_{c}-S\right)^{1 / \phi},
$$

where the critical exponent $\phi$ is equal to 2 (in the classical limit $\phi=1) .{ }^{3}$ In Eq. (24), $S_{c}$ is the value of the interaction parameter at $T_{c}=0 .{ }^{23,24}$ Thus, from Eqs. (9) and (24) through Eq. (20), one finds

$$
\gamma_{p}=\gamma_{T} / \phi
$$

which is equal to 1 at $T_{c}=0$ according to the quantum theory.

This gives $\gamma_{T}=2$ in the quantum limit and $\gamma_{T}=1$ in the classical limit, as stated above. Therefore, we conclude on the basis of the $\gamma_{p}=1$ value $^{3}$ that our value of $\beta_{p}=1 / 2$ for the spontaneous polarization is valid in the quantum limit $\left(T_{c}=0\right)$ and also at constant temperatures of 100 and $200 \mathrm{~K}$ in the cubic phase of $\mathrm{BaTiO}_{3}$.

\section{Conclusions}

Critical behavior of the dielectric susceptibility and the spontaneous polarization was analyzed for the ferroelectricparaelectric phase transition in $\mathrm{BaTiO}_{3}$. This analysis was employed on the basis of the Landau mean field theory and from the values of the critical exponent $\gamma$ for the dielectric susceptibility, the $\beta$ values for the spontaneous polarization were extracted according to $\beta=\gamma / 2$ between the classical and quantum limits for the ferroelectric-paraelectric transition in $\mathrm{BaTiO}_{3}$.

Similar method of analysis can be done for some other ferroelectric materials which undergo successive phase transitions such as $\mathrm{BaTiO}_{3}$.

\section{References}

${ }^{1}$ F. Jona and G. Shirane, Ferroelectric Crystals, Chapters 4 and 5 (The Macmillan Company, New York, 1962).

${ }^{2}$ G. A. Samara, Pressure and temperature dependences of the dielectric properties of the perovskites $\mathrm{BaTiO}_{3}$ and $\mathrm{SrTiO}_{3}$, Phys. Rev. 151, 378 (1966).

${ }^{3}$ T. Ishidate, S. Abe, H. Takahashi and N. Mori, Phase diagram of $\mathrm{BaTiO}_{3}$, Phys. Rev. Lett. 78, 2397 (1997).
${ }^{4}$ Y. Akishige, H. Takahashi, N. Mori and E. Sawaguchi, Vanishing of the phase transitions in ferroelectric hexagonal $\mathrm{BaTiO}_{3}$ at high pressure, J. Phys. Soc. Jpn. 63, 1590 (1994).

${ }^{5}$ Y. Akishige, T. Atake, Y. Saitoh and E. Sawaguchi, Specific heat of hexagonal barium titanate, J. Phys. Soc. Jpn. 57, 718 (1988).

${ }^{6} \mathrm{D}$. Heiman and S. Ushioda, Dispersion of the soft E-mode polariton in $\mathrm{BaTiO}_{3}$, Phys. Rev. 9, 2122 (1974).

${ }^{7}$ Y. Tomihaga and T. Nakamura, Temperature dependence of dispersion relation of over-damped E-polariton in $\mathrm{BaTiO}_{3}, J$. Phys. Soc. Jpn. 39, 746 (1975).

${ }^{8}$ A. Scalabria, A. S. Chaves, D. S. Shim and S. P. S. Porto, Temperature dependence of the $A_{1}$ and $\mathrm{E}$ optical phonons in $\mathrm{BaTiO}_{3}$, Phys. Stat. Sol. B 79, 731 (1977).

${ }^{9}$ J. L. Verble, E. Gallego-Lluesma and S. P. S. Porto, First-order $A_{1}$ (TO) phonons as evidence of disorder in $\mathrm{BaTiO}_{3}$, J. Raman Spectrosc. 7, 7 (1978).

${ }^{10}$ Y. Luspin, J. L. Servoin and F. Gervais, Soft mode spectroscopy in barium titanate, J. Phys. C: Solid State Phys. 13, 3761 (1980).

${ }^{11}$ K. Inoue, A. Hasegawa, K. Watanabe, H. Yamaguchi, H. Uwe and T. Sakudo, Silent soft mode in hexagonal barium titanate observed by hyper-Raman scattering, Phys. Rev. B 38, 6352 (1988).

${ }^{12}$ M. Yamaguchi, M. Watanabe, K. Inoue, Y. Akishige and T. Yagi, Light scattering study of the coupled soft-optic and acoustic mode in hexagonal barium titanate, Phys. Rev. Lett. 75, 1399 (1995).

${ }^{13} \mathrm{~S}$. Gupta, Investigations of micro-stress and phase transition in sol-gel-derived multideposited coatings of barium titanate using Raman spectroscopy, J. Raman Spectrosc. 33, 42 (2002).

${ }^{14}$ M. El Marssi, F. Le Marrec, I. A. Lukyanchuk and M. G. Karkut, Ferroelectric phase in barium titanate epitaxial thin film, Ferroelectrics 291, 55 (2003).

${ }^{15} \mathrm{P}$. Sedykh and D. Michel, Ferroelectric phase transition in barium titanate nanoparticles, Phys. Rev. B 79, 134119 (2009).

${ }^{16}$ A. Kiraci and H. Yurtseven, Temperature dependence of the Raman frequency, damping constant and the activation energy of a soft-optic mode in ferroelectric barium titanate, Ferroelectrics 432, 14 (2012).

${ }^{17} \mathrm{H}$. Yurtseven and A. Kiraci, Calculation of the damping constant and the relaxation time for the soft-optic and acoustic mode in hexagonal barium titanate, Ferroelectrics 437, 137 (2012).

${ }^{18}$ A. Kiraci and H. Yurtseven, Damping constant calculated as a function of temperature for the tetragonal Raman mode close to the paraelectric-ferroelectric transition in $\mathrm{BaTiO}_{3}$, Ferroelectrics 450, 93 (2013).

${ }^{19}$ W. Heywang, J. Am. Cer. Soc. 47, 484 (1964).

${ }^{20}$ K. H. Cho and H. Y. Lee, Pore dependent dielectric and electrical properties of barrium titanate ceramic, Proc. IEEE (1995) 566.

${ }^{21}$ S. Yasmin, S. Choudhury, M. A. Hakim, A. H. Bhuiyan and M. J. Rahman, structural and dielectric properties of pure and cerium doped barium titanate, J. Cer. Proc. Res. 12, 387.

${ }^{22} \mathrm{H}$. Yurtseven and A. Kiraci, Temperature dependence of the polarization and dielectric constant near the paraelectric-ferroelectric transition in $\mathrm{BaTiO}_{3}$, J. Mol. Model. 19, 3925 (2013).

${ }^{23}$ T. Schneider, H. Beck and E. Stoll, Phys. Rev. B 13, 1123 (1976).

${ }^{24}$ R. Morf, T. Schneider and E. Stoll, Phys. Rev. B 16, 462 (1977). 